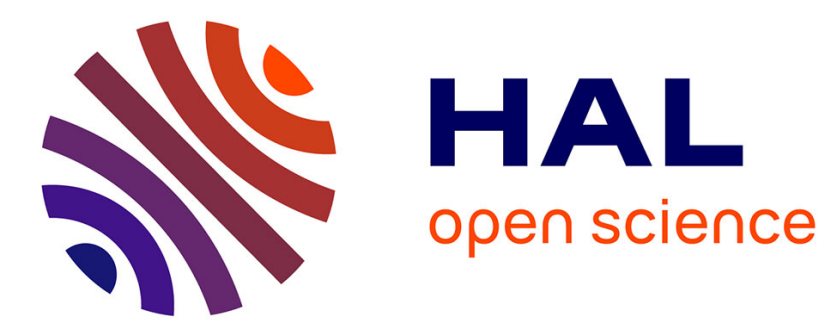

\title{
Fostering the Link from PLM to ERP via BIM \\ Dominik Holzer
}

\section{To cite this version:}

Dominik Holzer. Fostering the Link from PLM to ERP via BIM. 11th IFIP International Conference on Product Lifecycle Management (PLM), Jul 2014, Yokohama, Japan. pp.75-82, 10.1007/978-3-66245937-9_8. hal-01386477

\section{HAL Id: hal-01386477 \\ https://inria.hal.science/hal-01386477}

Submitted on 24 Oct 2016

HAL is a multi-disciplinary open access archive for the deposit and dissemination of scientific research documents, whether they are published or not. The documents may come from teaching and research institutions in France or abroad, or from public or private research centers.
L'archive ouverte pluridisciplinaire HAL, est destinée au dépôt et à la diffusion de documents scientifiques de niveau recherche, publiés ou non, émanant des établissements d'enseignement et de recherche français ou étrangers, des laboratoires publics ou privés. 


\title{
Fostering the Link from PLM to ERP via BIM
}

\section{The AEC Industry in Transition}

\author{
Dominik Holzer
}

The University of Melbourne, AUSTRALIA

\begin{abstract}
This paper investigates approaches for linking Product Lifecycle Management (PLM) via structured data stemming from Building Information Modeling (BIM) to information systems that get applied for Enterprise Resource planning (ERP) across Architecture, Engineering and Construction (AEC). The author highlights key pathways for such integration, with a particular focus on the hurdles contractors, suppliers and manufacturers need to overcome to master their transition to BIM-enabled PLM and the associated ERP. Based on a case-study (Hickory Group), the paper analyses the opportunities for the strategic repositioning of a construction and manufacturing firm who combines PLM with BIM and ERP within its organisation.
\end{abstract}

\section{Background to the PLM-ERP integration}

Expanding from product development and lifecycle planning into resource management is an approach that has steadily gained momentum since the introduction of ERP systems in the early 70s [1]. As a production-oriented sector, manufacturing benefits in particular from PLM-ERP linkages and consequently the use of well integrated information systems. Such systems assist the management of a broad range of processes relating to the product lifecycle. Similar to PLM, there is a number of core-activities specific to ERP. Being more transaction-oriented, these activities cover tasks such as resource planning, purchasing, storage, QA, change management, productivity management, HR, finances/payroll, and more. Common to most ERP systems (and similar to PLM) is the integration and management of data via a centralised server in a standardised format, in order to avoid 
data-duplication. With the progressing development of the World Wide Web, systems for ERP are increasingly becoming location agnostic, web-based, with information stored and accessed via cloud-based services [2]. If integration between PLM and ERP is desired, it is crucial for any organisation to create logic connections between product structure and production planning. A clear mapping of processes and points of connection is required to create bi-directional links between the two activities. Often the common point of connection is the Bill-Of-Materials (BOM) [3].

A key obstacle to the introduction of ERP to the construction industry can be highlighted as follows [4]: ERP systems provide scalable solutions that ideally help to manage information across an enterprise in its totality. On the other hand, the construction industry (and the support systems applied therein) is highly projectbased. A large number of external stakeholders federate information on a temporary (project-dependent) level, using a number of disparate information systems and formats. Establishing ERP systems in construction firms is therefore a task that needs to be considered specific to a highly dynamic project and productionbased context [5]. Also, the effort for implementing PLM and ERP solutions in an organisation is difficult to justify in smaller or medium sized organisations (which are common in the construction sector)

\section{BIM as an enabler}

In the past, efforts have been undertaken to link construction information and manufacturing data for construction to ERP systems. These efforts were mainly based on CAD output for documentation with added data/attributes to $2 \mathrm{D}$ documents. The proportion of valuable information-content that can easily be made available from 2D CAD to ERP is low. Consequently the use of 2D CAD is not best suited to tie in with the information-centric approach required to establish a clearly defined product structure. Research has been undertaken how to better manage the integration of product information in the construction process [6]. BIM provides such integration and its inclusion into planning and construction processes seems to promise increased connectivity between PLM and ERP [7].

It has now been over 10 years since BIM became officially accepted as the acronym across the construction industry to represent the breadth of approaches for object-based virtual modelling and building product models to encompass the entire building lifecycle. Up until that point (in 2003), variations of BIM had been applied across the construction industry globally in one way or another for 2-3 
decades. By 2014, the adoption of BIM methods for the design and delivery of projects has increased substantially [8]. BIM is becoming ever more mainstream, driven by a number of key countries such as the US, UK, Singapore, Finland, Denmark, Norway, and Australia [8]. Many other nations follow this international trend where more and more developed countries are becoming knowledgeable in the implementation and application of BIM related processes, policies, training, and procurement. An ever growing number of construction sectors globally embrace the added benefits of a more integrated and streamlined way of operation ${ }^{1}$.

Initially architecture and engineering firms were the first to adopt BIM to strengthen their in-house (lonely BIM) design/analyse/delivery methods. By nature, consultants operating with BIM tend to create 'Design Intent' BIM $^{2}$ (or LOD $^{3}$ 200/300) that serve design coordination without necessarily involving a description of the detailed object to be installed by the trades. This approach has been complemented over the past few years with the production of 'Construction BIM' (LOD 400) as more and more contractors embrace BIM for detailed coordination and quantity take-off during construction and assembly. In order to achieve the required level of detail, contractors work with sub-contractors and suppliers who provide coordinated BIM that consider actual manufacturing for detailed assembly and the extraction of Bill-of-Quantities of actual equipment components. By incorporating coordinated object data from sub-contractors and suppliers into their delivery process, the construction industry moves towards system integration and process automation in an unprecedented way. The link from BIM data output to fabrication equipment either directly, or via a CAD/CAM interface is becoming more commonplace throughout a number of trades within the construction industry. Pre-fabrication and offsite assembly is on the rise globally.

The use of BIM is expanding with progressive convergence between information systems that logically tie into the data-rich environment it offers. In order to achieve data-integrity, the construction industry increasingly relies on standardised formats to structure BIM object data via Integrated Construction Information

\footnotetext{
${ }^{1}$ McGraw Hill has issued a smart market report on: "The Business Value of BIM for Construction in Major Global Markets" in early 2014. The report outlines in detail how the construction sector in various developed markets globally has taken up BIM.

${ }^{2}$ The Consensusdocs 301 - BIM Addendum makes a clear distinction between design intent and construction BIM. It is the first standard contract document that globally addresses legal and administration issues associated with using BIM.

${ }^{3}$ The American Institute of Architects (AIA) 'E202-2008 BIM Protocol' was the first document to classify the progression of BIM into five major stages of development (LOD $100-500$ ) ranging from conceptual modelling to construction and operation.
} 
Systems (ICIS) [4]. On one end of the spectrum, BIM increasingly gets linked to geospatial data and GIS integration for City Information Modeling. BIM now plays an important role as a stepping stone to connect geometric information and spatial data from the building level, to a 'precinct' scale, and finally the overarching geospatial context (GIS). On the other end of the spectrum, BIM methods get applied for manufacturing and detailed assembly of construction components. On this component level, the construction industry (finally) comes to terms with the relevance of managing highly detailed virtual representations of the built artefacts. An increasing number of BIM objects are available via libraries and manufacturer/reseller's websites. Hand in hand with this development, BIM encompasses an ever larger number of stakeholders throughout the building lifecycle (social BIM) - in line with the initial ideals by its proponents [9].

\section{The Relation between BIM, PLM and ERP}

Despite a number of similarities, investigations into the PLM to ERP transition has rather occurred in parallel to (and not in conjunction with) the development of BIM over the past 3+ decades [5]. A key point of investigation therefore relates to the question of how to establish authoritative data that allows organisations to communicate between all three systems. Resolving the definition of authoritative data that cuts across product lifecycle, resource planning and building information modeling processes may lead to highly efficient sharing of data across these processes. Such integration would likely result in an increase of efficiency throughout a number of inter and intra-organisational business processes.

Only recently BIM has matured to a point where the construction industry starts to explore closer links between BIM, PLM and ERP on a mainstream level. There are four main reasons for this delay:

Firstly: detailed coordination of virtual building objects for construction is fairly new to the AEC industry (apart from a few exceptions) where subcontractors and trades only slowly start to embrace BIM. A manufacturing mindset is not always prevailing in the construction industry that is historically rooted in craft-based skills and the experience of its workers.

Secondly: the construction industry needs to overcome the hurdle of setting up and adopting protocols that enable interoperability and integrated data-sharing across a number of stakeholders. 
Thirdly: Software linking data from object-oriented BIM assemblies directly to ERP systems has only recently become mature and available to the mainstream construction market.

Fourthly: Many firms in the AEC industry are small or medium in size [11]. Whereas BIM use has become common in these firms, the effort to implement PLM or ERP systems across those firms may seem too costly and undesirable

Still, many goals of BIM have strong overlap with the goals of both PLM and ERP. The application of BIM on construction projects aims at streamlining the information flow across the building lifecycle using high-fidelity data associated to virtual building components that represent their physical counterparts as closely as possible. Despite their traditionally different scope of service, there are manifold possibilities for connecting product data from design and engineering to PLM [12]. Agram and Eastman list the following five key areas of connection: System Configuration, Authoring Information, General Management Functions, Change Management \& Synchronisation, and Data Visualization \& Interoperability.

In the context of BIM, key to the integration between PLM and BIM is the ability to synchronise data between the PLM system and the BIM server, based on the definition of authoritative data. In order to achieve such an interface between BIM and PLM systems a logic for the creation, naming, tagging and management of object data (and the opportunities to interface this information with other serverbased systems) has to be established across design and planning within an organisation. BIM objects include, category, type, and attribute definitions; parametric relations can be established to govern these definitions across the entire buildinglifecycle and help optimise sustainable building design [9].

Other industries such as car-manufacturing or aerospace have long experienced the benefits of PLM and ERP use within their associated manufacturing sectors [10]. It is therefore no surprise that the first steps for integrating BIM with PLM and further with ERP in construction were facilitated by software environments that can also be found in parallel industries. Dassault Systèmes is a company that has steadily expanded such environments from industries like Aerospace and Financial Services to facilitate solutions within Architecture, Engineering and Construction. Their Logistics offering includes equipment placing, human modelling $\&$ simulation, material handling and flow to the site, cost \& construction time estimation, just to name a few [13]. In 2008, Skanska was one of the first organisations to employ Dassault's Enovia ${ }^{\text {TM }}$ PLM solution in conjunction with their BIM approach and the goals to further integrate it with ERP. Skanska has since done so 
for data management across the supply chain on a number of their projects building on their PLM approach to establish authoritative data.

\section{The Hickory Example}

Most contractors and manufacturers that operate in the construction industry, have yet to explore the PLM/BIM/ERP integration [9] [14]. Those organisations that actively pursue a path of integration between PLM, BIM and ERP often see themselves entering unchartered territory.

In an attempt to draw from a practical example of PLM, BIM and ERP integration, the author has conducted research at one organisation in particular that actively promotes their supply-chain integration in the production of unitised building systems. The Victoria-based enterprise surrounding the Hickory Group is on the forefront of innovation and development in the Australian construction sector. Founded in 1991, Hickory combines a construction business with manufacture, plant hire, and more. Next to their conventional construction division, the Hickory Group oversees a division for prefabrication and assembly of building units (UB), as well as a division for the pre-fabrication bathroom pods as specialised building systems (SYNC). What distinguishes Hickory from most of their competitors in Australia is their strong focus on streamlining the fabrication processes of their production line, applying a manufacturing mindset rather than construction. This approach becomes most apparent within their UB and SYNC divisions where they collaborate with designers on new patents and innovative production methods. Over a period of 1 year, the author has conducted two site visits to the Hickory production facilities where he inspected the production line as well as interviewing Hickory staff, who form part of their drafting team, their process management group, their production management group, as well as the organisation's leadership.

Whereas BIM is based on disruptive technology for the delivery of design documentation and beyond, a transition to an ERP system affords the integration of highly disruptive technology to all sectors of their business. Challenges faced by Hickory on their path of embracing PLM and ERP relate to the fundamental changes required on their path to gain benefits from an introduction of related systems to their business. Segregated business units within the Hickory group were re-integrated in order to streamline data transfer across IT systems and management practices across different sectors of the enterprise. Such a move also allowed 
for increased standardisation naming conventions related to their resource planning and production processes.

Initiated by new leadership, Hickory recently expanded their competencies by employing new staff with manufacturing background. In addition, external consultants assist Hickory with a major overhaul of their internal project delivery processes. The overhaul goes hand in hand with the establishment of a new datamanagement strategy that covers the integration of PLM with ERP and BIM. The shift from a construction to a manufacture is manifest in a number of ways:

- A strong focus on frontloading the design effort in order to identify and validate detailed assembly requirements as early as possible

- Logical naming conventions that allow for fluent data transfer between PLM, BIM and ERP

- Knowledge engineering based on detailed analysis of prototypes and knowledge transfer further down the supply-chain

- A revised tool ecology with detailed specification of data-transfer and management.

- Introducing detailed process plans with clearly defined hold-points, complemented with progress checklists and identification of high-risk items.

- Consolidating separate information systems across the entire business into a centralised system for data storage and management with particular focus on the integration between PLM, ERP and BIM.

Key to the above activities is the establishment and assignment of authoritative ('master') data to those processes where most of the information is required. This approach involves upfront planning via PLM with the consequence that design changes can be traced and communicated directly to the BOM for production planning via their ERP (and in particular their Material Resource Planning MRP). At the UB and SYNC divisions at Hickory, Autodesk's PLM solution Vault ${ }^{\mathrm{TM}}$ was chosen to facilitate this transition and manage the 'master' data. A third party technology consultancy was commissioned to revise the existing tool ecology and process map across the Hickory group. The consultancy established an implementation strategy in order to enable the streamlined integration of virtual 3D geometric construction objects in LOD 400 BIM (shop-drawing) level, their associated data for construction, and the organisation's ERP systems that ties the $\mathrm{BIM} / \mathrm{PLM}$ data to the production process. The increasing information content incorporated into the 3D BIM for assembly provides the backdrop for data integration in Vault ${ }^{\mathrm{TM}}$. In order to streamline the transfer from BIM to the PLM system, Hickory has transitioned from using Autodesk's Revit ${ }^{\mathrm{TM}}$ to Inventor ${ }^{\mathrm{TM}}$ as their predominant documentation tool. It was identified that Revit ${ }^{\mathrm{TM}}$ 's integration with 
Vault $^{\mathrm{TM}}$ is insufficiently resolved to facilitate the level of authoritative data integration aspired to by Hickory. Inventor ${ }^{\mathrm{TM}}$ on the other hand has a stronger manufacturing focus than Revit ${ }^{\mathrm{TM}}$, with better integration to Vault ${ }^{\mathrm{TM}}$. BIM drafters at Hickory had to be up-skilled and new staff was hired in order to accommodate the revised workflow.

Risk analysis at UB and SYNC revealed that linking between ERP and BIM could not be achieved successfully on the first available project. Instead, UB at Hickory are gradually migrating their data storage and management from a spreadsheetbased approach to a centralised server, increasing the information content, value engineering, and data management ${ }^{4}$ capabilities as they go. The Hickory team is gradually learning to adjust and refine the interfaces between the lifecycle planning and production processes, but there are great expectations for the streamlined interface to deliver major benefits to the business once major steps in the implementation have been accomplished.

\section{Future Goals}

The transition such as seen at Hickory exemplifies a major shift in thinking by local construction companies: The ultimate goal for integrating PLM with BIM and ERP does not merely lie in the streamlining of processes and the reduction of production/business cost. Much more than that, if lifecycle management, resource planning, and design can be connected via robust processes and high-fidelity data, a company could then export that 'system' and manufacture anywhere in the world. Design will need to be rationalised to a point where it can be realised through knowledge-engineering and related manufacturing processes. Mastering such an approach will allow a construction firm to positions itself as strong competitor on a global market, dramatically reducing production cost, increasing turnover of capital, and becoming manufacturers rather than remaining builders.

${ }^{4}$ Such as Kanban systems and change-tracking to increase automation 


\section{Conclusions}

After 10 years of BIM use across the construction industry undergoes increasing convergence due to the efforts by various professions to establish the closest possible match between virtual representations of building components and their physical counterpart. BIM is now steadily expanding into other areas and its use has reached a level of maturity where data fidelity is sufficiently resolved that BIM output can interact with PLM systems and further drive the definition of Bills-Of-Material that feed into ERP processes and help to drive the production line.

The construction industry is still in early days of exploring the full potential of these connections. Any organisation aiming to make a transition towards greater synergies between their planning and production process using BIM, PLM and ERP, needs to account for a possible temporary drop in productivity. Re-skilling of staff (and likely also the employment of experts from other industry sectors), the re-organisation of established intra and inter-organisation processes, data integration between business sectors, and a revised tool infrastructure are just a few points in a long list of challenges such organisations are facing. Changing the mentality from a traditional mindset (that is at times still rooted in a craft-based industry) towards highly engineered manufacturing and production does not occur overnight.

Despite the obstacles, those organisations within the construction industry who innovate with a strong focus on supply-chain integration, pre-fabrication, rapid manufacture and assembly, have most to gain by embracing the possibilities for tight links between BIM, PLM and ERP. They will be able to position themselves as strong global players on a competitive market that will increasingly rely on manufacturing and automation. 


\section{Acknowledgments}

The author would like to acknowledge the assistance from the Hickory Group as well as Memko in the development of this paper.

\section{References}

[1] Fitzgerald, A. (1992) “Enterprise Resource Planning”. IEE Conference Publication 359, Institute of Electrical Engineers, London, UK, 291-297

[2] Wilkinson, P. Online Resource: "Unit4 combining collaboration, ERP and BIM in the cloud" http://www.extranetevolution.com/2012/02/unit4-combining-collaboration-erp-and-bim-inthe-cloud/\#sthash.dmlBT4ez.dpuf Accessed on 16.02.2014

[3] Shilovitsky, O., "BOM: Apple of Discord between PLM and ERP?" Online Resource: http://beyondplm.com/2013/09/17/bom-apple-of-discord-between-plm-and-erp/, Accessed on 16.02.2014

[4] Mêda, P. and Sousa, H. (2012) "Towards Software Integration in the Construction Industry ERP and ICIS Case Study”. Proceedings of the CIB W78 2012:29th International Conference - Beirut, Lebanon, 17-19 October, 304-313

[5] Babič, N.Č., Podbreznik, P., Reboljêda, D. (2010) "Integrating resource production and construction using BIM". Automation in Construction 19, 539-543

[7] Technia PLM Newsletter, "The Construction industry in focus" Online Resource: http://www.technia.com/Section-News-Events/Newsletters/Nordic-PLM-Newsletter/Issue-120102/Jonas-Chronical/The-Construction-industry-in-focus/ Accessed on 15.02.2014

[6] Cutting-Decelle, A.-F., Dubois, A.-M. and Fernandez, I. (1997) "Management and Integration of Product Information in Construction: Reality and Future Trends" The Int. Journal of Construction IT 5(2), pp. 19-46

[8] McGraw Hill (2014), "SmartMarket Report, The Business Value of BIM for Construction in Major Global Markets", How Contractors around the world are driving innovation with Building Information Modeling, McGraw Hill Construction

[9] Eastman, C. (1999) "Building Product Models: Computer Environments Supporting Design and Construction", Boca Raton, Fla. London, CRC Press

[10] Cimalore, C. (2007) "ERP/PLM complement each other \& facilitate a collaborative environment in design, engineering and manufacturing processes." Electronics Supply Manufacturing

[11] Ghosh, S., Negahban, S., Kwak, J.H. and Skibniewski, M.J. (2011) "Integration of PLM Solutions and BIM Systems for the AEC Industry". Proceedings of the 30th ISARC, Montréal, Canada, 1046-1055

[12] Aram, S. and Eastman, C. (2013) "Impact of Sustainability on Integration and Interoperability between BIM and ERP - A Governance Framework". Proceedings of the Technology Management Conference (ITMC), 2011 IEEE International, 187-193

[13] Dassault Systèmes: Logistics, Online Resource http://www.3ds.com/industries/architectureengineering-construction/business-processes/logistics/, Accessed on 16.02.2014

[14] Barthorpe, S., Chien, H. J., \& Shih, J. K. (2004). A survey of the potential for enterprise resource planning (ERP) in improving the effectiveness of construction management in the UK construction industry. International journal of computer applications in technology, 20(1), $120-128$. 